Bulletin of the Section of Logic

Volume 51/1 (2022), pp. 1-26

https://doi.org/10.18778/0138-0680.2021.26

Theofanis Aravanis

\title{
AN EPISTEMOLOGICAL STUDY OF THEORY CHANGE
}

\begin{abstract}
Belief Revision is a well-established field of research that deals with how agents rationally change their minds in the face of new information. The milestone of Belief Revision is a general and versatile formal framework introduced by Alchourrón, Gärdenfors and Makinson, known as the AGM paradigm, which has been, to this date, the dominant model within the field. A main shortcoming of the AGM paradigm, as originally proposed, is its lack of any guidelines for relevant change. To remedy this weakness, Parikh proposed a relevance-sensitive axiom, which applies on splittable theories; i.e., theories that can be divided into syntax-disjoint compartments. The aim of this article is to provide an epistemological interpretation of the dynamics (revision) of splittable theories, from the perspective of Kuhn's influential work on the evolution of scientific knowledge, through the consideration of principal belief-change scenarios. The whole study establishes a conceptual bridge between rational belief revision and traditional philosophy of science, which sheds light on the application of formal epistemological tools on the dynamics of knowledge.
\end{abstract}

Keywords: Belief revision, epistemology, Parikh, relevance, Kuhn, scientific knowledge.

2010 Mathematical Subject Classification: 03B42, 68T27.

\section{Introduction}

A well-established research field that lies at the intersection of Formal Philosophy and Computer Science, and deals with how agents rationally

Presented by: Andrzej Indrzejczak

Received: October 9, 2020

Published online: November 9, 2021

(C) Copyright by Author(s), Łódź 2022

(C) Copyright for this edition by Uniwersytet Łódzki, Łódź 2022 
change their minds in the face of new information, is that of Belief Revision $[14,24,13] .{ }^{1}$ Roughly speaking, the process of belief revision can be outlined as follows [14, 24]:

- A rational agent receives new information (epistemic input).

- In the principal case where the new information contradicts her initial beliefs, the agent needs to withdraw some of the old beliefs before she can (consistently) accommodate the new information.

- The agent is, also, obliged to accept the consequences that might result from the interaction of the new information with the (remaining) old beliefs.

What makes the problem non-trivial is that several different ways for performing the revision-process may be possible. Suppose, for example, that the beliefs of a rational agent are composed of the following three propositions:

i) All African lions are brown.

ii) The animal Bob encounters is a lion.

iii) The animal Bob encounters comes from Africa.

Along with the above three propositions, the agent is obliged to believe their following immediate consequence:

iv) The animal Bob encounters is brown.

Suppose, now, that the animal Bob encounters turns out to be white. In order for the agent to maintain a consistent corpus of beliefs after adding the fact about lion's whiteness, she needs to revise her initial beliefs. That is to say, some of her original beliefs must be withdrawn. Clearly, she does not want to give up all her beliefs, since this would be an unnecessary loss of valuable information. It is not hard to verify that, in the case described above, there are at least three different ways for performing revision. In general, this can be done in a number of ways. More importantly, the problem of belief revision is that logical considerations alone are not sufficient

${ }^{1}$ To distinguish the research area from the process, we shall use the capitalized term Belief Revision for the former, and the lower-case term belief revision for the latter. 
for choosing which beliefs have to be given up; this has to be decided by means of extra-logical structures.

The benchmark of Belief Revision is a general and versatile formal framework introduced by Alchourrón, Gärdenfors and Makinson, known as the AGM paradigm (after the initials of its originators), which has been, to this date, the dominant model within the field [1]. The AGM paradigm captures both axiomatically and constructively the process of rational belief revision. Axiomatically, by means of rationality postulates that any rational revision function ought to satisfy, and constructively, by means of extra-logical structures based on preference orderings.

A main shortcoming of the AGM paradigm, as originally introduced, is its insufficiency to capture the notion of relevance. To remedy this weakness, Parikh proposed a postulate that supplements the approach of Alchourrón, Gärdenfors and Makinson; the postulate essentially captures a form of syntactical relevance, and is typically referred to as axiom $(\mathrm{P})$ [23]. Roughly speaking, axiom (P) states that the revision of a splittable theory $K$-i.e., a theory that can be divided into syntax-disjoint compartments referring to mutually irrelevant subject matters - by an epistemic input which (syntactically) relates only to some compartment of $K$, should not affect any other compartment of $K$. A central concept that Parikh used for developing his axiom is that of theory-splitting [23].

In the present article, we discuss the revision of splittable theories, through the prism of the influential 1970 book "The Structure of Scientific Revolutions" by Thomas Kuhn, which studies the evolution of scientific knowledge [21]. The task is accomplished by considering a Kuhnian reading of principal belief-change scenarios involving Parikh's concept of theory-splitting; essentially, we argue that these scenarios can be regarded as a reflection of notable phases of scientific development. Our aim is the establishment of a conceptual bridge between rational belief revision and traditional philosophy of science, which will shed light on the application of formal epistemological tools on the dynamics of (any corpus of) knowledge. $^{2}$

The remainder of this article is structured as follows. The next section provides a brief overview of Kuhn's work on philosophy of science. Thereafter, Section 3 sets the formal background for our discussion, followed by

\footnotetext{
${ }^{2}$ For an excellent book that discusses a variety of perspectives concerning belief revision in the context of scientific enquiry, the interested reader is referred to [22].
} 
Section 4 which presents the AGM paradigm. Section 5 introduces Parikh's notion of relevance, as well as some fundamental definitions based on this notion. Section 6 discusses the revision of splittable theories from an epistemological perspective, and Section 7 reports a collection of representative examples from the history of science that justify the conducted study. A brief concluding section closes the article.

\section{Kuhnian epistemology in a nutshell}

According to Kuhn, the phases of scientific progress can be summarized as follows [21]:

- Pre-paradigm period.

- Normal science.

- Crisis.

- Scientific revolution.

- New normal science.

- New crisis.

Prior to the formation of a shared paradigm or research consensus (preparadigm period), would-be scientists are devoted to the accumulation of random facts and unverified observations. This non-organized activity acquires coherence once the scientific community adopts a unique shared paradigm. A paradigm is a distinct set of concepts or thought patterns (including theories and research methodologies), that constitute legitimate contributions to a field. The paradigm is adopted jointly by the members of a scientific community, it establishes the (nature of the) entities of the world (i.e., an ontological acceptance), as well as a common language.

Everyone working within a settled paradigm (e.g., Classical Mechanics) is doing normal science. In the context of normal science - which Kuhn describes as a puzzle-solving process - scientists are slowly accumulating details in accord with an established broad theory, making the paradigm more coherent and concrete. During this process, the scientific community does not question or challenge the underlying (philosophical and metaphysical) assumptions of the theory. 
During the period of normal science, the observable new information (epistemic input) is formulated within the language of a particular theory, and its validity depends on the validity of the corresponding theoretical or conceptual context. In this sense, theories precede observations. Theories can be formulated - and this is usually the case - prior to the observations that contribute to their justification. Generally, the meaning of a concept is (at least partially) "sculptured" from the role it plays in a theory.

For the normal scientist, anomalies represent challenges to be puzzled out and solved within the settled paradigm. At the point where such anomalies cannot be handled within the paradigm, a crisis emerges. In case an anomaly (or series of anomalies) persists long enough, and for enough members of the scientific community, the paradigm will itself gradually come under challenge, and perhaps be subjected to a paradigm shift, a process often also described as a scientific revolution. After the scientific revolution, a new paradigm is established by the majority of the community, which takes the place of the old problematic one, and a period of new normal science begins. ${ }^{3}$ It is noteworthy that, according to Kuhn, the language and theories of successive paradigms are incommensurable, in the sense that, in principle, they cannot be translated into one another, or rationally evaluated against one another, by means of a formal framework. Kuhn argues that incommensurability constitutes a universal property of scientific revolutions.

Having presented the core principles of Kuhn's work on the evolution of scientific knowledge, we turn to a more analytical tone.

\section{Formal background}

Throughout this article, we shall be working with a propositional language $\mathcal{L}$, built over a finite, non-empty set $\mathcal{P}$ of propositional variables (atoms), using the standard Boolean connectives $\wedge$ (conjunction), $\vee$ (disjunction), $\rightarrow$ (implication), $\leftrightarrow$ (equivalence), $\neg$ (negation), and governed by classical propositional logic. ${ }^{4}$ This abstraction is made, mainly, due to the fact that the majority of belief-revision studies, including Parikh's exposition in [23],

\footnotetext{
${ }^{3}$ Two representative cases of paradigm shifts are the transition from a Ptolemaic Cosmology to a Copernican one, as well as the acceptance of plate-tectonics theory (replacing the idea of continental drift) as an explanation for large-scale geological transformations.

${ }^{4}$ Notice that no zero-ary connectives are considered.
} 
are conducted assuming classical propositional logic, a fact which, in turn, provides easier presentation. Another argument in favour of this convenient formalism, is that, in the context of Answer Set Programming (ASP) [11], which constitutes a contemporary formal framework used for modelling the dynamics of a plethora of real-world scientific domains, although a particular scenario is modelled in the syntax of first-order logic, the system ultimately solves a finite propositional representation of it (produced through a sophisticated process called grounding). We note, lastly, that, even if the essence of our approach easily extends to richer formalisms, a formal account of this issue would be an interesting avenue for future investigation.

A sentence of $\mathcal{L}$ is contingent iff it is neither a tautology nor a contradiction. For a set of sentences $\Gamma$ of $\mathcal{L}, C n(\Gamma)$ denotes the set of all logical consequences of $\Gamma$, i.e.,

$$
C n(\Gamma)=\{\varphi \in \mathcal{L}: \Gamma \models \varphi\},
$$

where $\models$ stands for the classical consequence relation. We shall write $C n\left(\varphi_{1}, \ldots, \varphi_{n}\right)$ for sentences $\varphi_{1}, \ldots, \varphi_{n}$, as an abbreviation of $C n\left(\left\{\varphi_{1}, \ldots, \varphi_{n}\right\}\right)$.

An agent's belief corpus shall be modelled by a theory, also referred to as a belief set. A theory $K$ is any deductively closed set of sentences of $\mathcal{L}$; i.e.,

$$
K=C n(K) .
$$

The set of all theories is denoted by $\mathbb{K}$. A theory $K$ is complete iff, for all sentences $\varphi \in \mathcal{L}$, either $\varphi \in K$ or $\neg \varphi \in K$. For a theory $K$ and a sentence $\varphi$ of $\mathcal{L}$, the expansion of $K$ by $\varphi$, denoted by $K+\varphi$, is the deductive closure of the set $K \cup\{\varphi\}$, i.e.,

$$
K+\varphi=C n(K \cup\{\varphi\}) .
$$

A literal is a propositional variable or its negation. A possible world (or, simply, world) $r$ is any consistent set of literals, such that, for any propositional variable $p \in \mathcal{P}$, either $p \in r$ or $\neg p \in r$. The set of all possible worlds is denoted by $\mathbb{M}$. For a sentence (or set of sentences) $\varphi$ of $\mathcal{L},[\varphi]$ is the set of worlds at which $\varphi$ is true. For a set of worlds $V \subseteq \mathbb{M}$, we denote by $t h(V)$ the set of all sentences satisfied by all worlds in $V$; if $V=\varnothing$, then vacuously $t h(V)=\mathcal{L}$. It is not hard to verify that $t h(V)$ is a (unique) theory. 


\section{The AGM paradigm}

Within the AGM paradigm [1], the process of belief revision is modelled as a (binary) function $*$, which maps a theory $K$ and a sentence $\varphi$ to a revised (new) theory $K * \varphi$. Rational revision functions, the so-called $A G M$ revision functions, respect the $A G M$ postulates for revision, listed below. ${ }^{5}$

$(\mathbf{K} * \mathbf{1}) \quad K * \varphi$ is a theory of $\mathcal{L}$.

$(\mathbf{K} * \mathbf{2}) \quad \varphi \in K * \varphi$.

$(\mathbf{K} * \mathbf{3}) \quad K * \varphi \subseteq K+\varphi$.

$(\mathbf{K} * \mathbf{4}) \quad$ If $\neg \varphi \notin K$, then $K+\varphi \subseteq K * \varphi$.

$(\mathbf{K} * \mathbf{5}) \quad K * \varphi$ is inconsistent iff $\varphi$ is inconsistent.

$(\mathbf{K} * \mathbf{6})$ If $C n(\varphi)=C n(\psi)$, then $K * \varphi=K * \psi$.

$(\mathbf{K} * \mathbf{7}) \quad K *(\varphi \wedge \psi) \subseteq(K * \varphi)+\psi$.

$(\mathbf{K} * \mathbf{8}) \quad$ If $\neg \psi \notin K * \varphi$, then $(K * \varphi)+\psi \subseteq K *(\varphi \wedge \psi)$.

The AGM postulates for revision do not suffice to uniquely specify the revised belief set $K * \varphi$, given $K$ and $\varphi$ alone; they simply intend to circumscribe the territory of all different rational ways of revising belief sets. For a unique specification of $K * \varphi$, appropriate extra-logical tools are required, the so-called constructive models for belief revision, the first of which has already been proposed in the seminal work of Alchourrón, Gärdenfors and Makinson [1]. Herein, our focus is on a popular constructive model introduced by Katsuno and Mendelzon, which is based on a special kind of total preorders over possible worlds, called faithful preorders [18].

Before discussing the faithful-preorders model, we first recall that a preorder over a set $V$ is any reflexive, transitive binary relation on $V$. A preorder $\preceq$ is called total iff, for all $r, r^{\prime} \in V, r \preceq r^{\prime}$ or $r^{\prime} \preceq r$. As usual, the strict part of $\preceq$ shall be denoted by $\prec$; namely, $r \prec r^{\prime}$ iff $r \preceq r^{\prime}$ and $r^{\prime} \npreceq r$. Also, $\min (V, \preceq)$ denotes the set of all $\preceq$-minimal elements of $V$; i.e.,

${ }^{5} \mathrm{~A}$ detailed discussion on the postulates $(K * 1)-(K * 8)$ can be found in [14, Section 3.3] or [24, Section 8.3.1]. It is, also, noteworthy that several concrete "off-the-shelf" revision operators that satisfy $(K * 1)-(K * 8)$ have been proposed in the literature; see, indicatively, [12, 26, 7, 4]. 


$$
\min (V, \preceq)=\left\{r \in V \text { : for all } r^{\prime} \in V, \text { if } r^{\prime} \preceq r, \text { then } r \preceq r^{\prime}\right\} \text {. }
$$

Definition 4.1 (Faithful Preorder, [18]). A total preorder $\preceq_{K}$ over $\mathbb{M}$ is faithful to a theory $K$ iff the $\preceq_{K}$-minimal worlds of $\mathbb{M}$ are those satisfying $K$; i.e., $\min \left(\mathbb{M}, \preceq_{K}\right)=[K]$.

Intuitively, a faithful preorder $\preceq_{K}$ encodes the comparative plausibility of all possible worlds of $\mathbb{M}$, with respect to theory $K$, so that the more plausible a world is, the lower it appears in the ordering $\preceq_{K}$.

Definition 4.2 (Faithful Assignment, [18]). A faithful assignment is a function that maps each theory $K$ of $\mathcal{L}$ to a total preorder $\preceq_{K}$ over $\mathbb{M}$, which is faithful to $K$.

Katsuno and Mendelzon proceed, then, to the following representation theorem.

THEOREM $4.3([18])$. A revision operator $*$ satisfies postulates $(K * 1)$ $(K * 8)$ iff there exists a faithful assignment that maps each theory $K$ of $\mathcal{L}$ to a total preorder $\preceq_{K}$ over $\mathbb{M}$, such that, for any sentence $\varphi \in \mathcal{L}$ :

$$
(\mathbf{F} *) \quad K * \varphi=\operatorname{th}\left(\min \left([\varphi], \preceq_{K}\right)\right) .
$$

Essentially, condition $(\mathrm{F} *)$ specifies the revised theory $K * \varphi$ as the theory corresponding to the most plausible (with respect to $K$ ) $\varphi$-worlds. For ease of presentation, we shall consider, in the course of this work, only the principal case of consistent belief sets and contingent epistemic input.

Gärdenfors and Makinson have introduced another well-known model for constructing AGM revision functions, equivalent to the model of Katsuno and Mendelzon, which is based on the notion of epistemic entrenchment [16]. A central aspect of Gärdenfors and Makinson's model is a particular type of total preorder over all beliefs of a theory $K$, called epistemic-entrenchment preorder, which encodes the relative epistemic values of all the sentences in $K$. An investigation by Peppas and Williams of the interconnections between the two aforementioned constructive models revealed that an epistemic-entrenchment preorder, associated with a theory $K$, suffices to fully specify a faithful preorder $\preceq_{K}[25$, Theorem 6.3]. On that premises, and given that the definition of an epistemic-entrenchment 
preorder does not require the relative epistemic values of sentences not belonging to $K$, the following remark is true.

Remark 4.4. Let $K$ be a theory of $\mathcal{L}$, and let $\preceq_{K}$ be a total preorder faithful to $K$. A rational agent does not need to explicitly provide the relative epistemic values of sentences not belonging to $K$ (non-beliefs), in order for $\preceq_{K}$ to be specified.

Before closing this section, we point out an interesting feature of AGM revision functions. In particular, Theorem 4.5, subsequently, shows that there exist AGM revision functions such that, if we "feed" them with the appropriate input, their output is always confined to a particular "island" of belief sets.

THEOREM 4.5. There exist an AGM revision function $*$ and a proper subset $\Theta$ of $\mathbb{K}$, such that, for any theory $K \in \Theta$ and any $\varphi \in \mathcal{L}, K * \varphi \in \Theta$.

Proof: Let $\Theta$ be the set of all complete theories of $\mathcal{L}$. Clearly, $\Theta \subset \mathbb{K}$. Let * be an AGM revision function such that it assigns (via condition $(\mathrm{F} *)$ ) to each theory $K \in \Theta$ the following $K$-faithful preorder $\preceq_{K}$ over $\mathbb{M}$ :

$$
\begin{array}{lllllll}
w & \prec_{K} & r_{1} & \prec_{K} & r_{2} & \prec_{K} & \ldots
\end{array}
$$

where $w$ is a world of $\mathbb{M}$ such that $[K]=\{w\}$, and $r_{1}, r_{2}, \ldots$ is any sequence of all worlds in $\mathbb{M}-\{w\} .{ }^{6}$ By the construction of $\preceq_{K}$, it follows that, for any sentence $\varphi \in \mathcal{L}$, the set $\min \left([\varphi], \preceq_{K}\right)$ is always a singleton; that is, the revised belief set $K * \varphi$ is always satisfied by exactly one world. ${ }^{7}$ Therefore, for any theory $K \in \Theta$ and any $\varphi \in \mathcal{L}, K * \varphi \in \Theta$.

The above result does not only show that there are AGM revision functions that could result in a form of "islanding"; it, also, shows that such functions could "trap" a rational agent into an "omniscience island", in the sense that, if the agent has an opinion about everything (thus, her belief corpus coincides with a complete theory), she will still have an opinion about everything, after any sequence of revisions. ${ }^{8}$

\footnotetext{
${ }^{6}$ For any complete theory $K$, the set $[K]$ is a singleton.

${ }^{7}$ The AGM revision function $*$ implements at theory $K$ a type of revision called maxichoice revision [1, 14].

${ }^{8}$ If $K$ is a theory that represents the agent's beliefs, any possible world in $[K]$ is perceived by the agent to be the "actual" world. Hence, the more the agent learns, the fewer possible worlds are compatible with her knowledge. On that premise, a complete theory expresses the beliefs of an omniscient agent.
} 


\section{Parikh's notion of relevance}

Convincing concrete examples have pointed out that the AGM postulates for revision are insufficient to capture the notion of relevance. For instance, the severe full-meet revision satisfies the AGM postulates for revision and, at the same time, it discards all prior beliefs of a belief set $K$ retaining only the epistemic input $\varphi$, in the principal case where $\varphi$ contradicts $K$ [1]. On that unsatisfactory premise, Parikh proposed a supplementary axiom, named $(\mathrm{P})$ and presented below, that encodes a form of syntactical relevance $[23] .^{9}$

(P) If $K=C n(x, y)$, where $x, y$ are sentences of disjoint sublanguages $\mathcal{L}_{x}, \mathcal{L}_{y}$, respectively, and $\mathcal{L}_{\varphi} \subseteq \mathcal{L}_{x}$, then $K * \varphi=\left(C n_{\mathcal{L}_{x}}(x) \diamond \varphi\right)+y$, where $\diamond$ is a local revision operator defined over the sublanguage $\mathcal{L}_{x}$.

Some remarks on the notation in $(\mathrm{P})$ are in order. For a sentence $x$ of $\mathcal{L}$, $\mathcal{L}_{x}$ denotes the (unique) minimal (sub)language of $\mathcal{L}$ within which $x$ can be expressed; in the limiting case where $x$ is not contingent, $\mathcal{L}_{x}$ is defined to be the empty set. We note that this definition can be extended to a belief set $K$, since, given that $\mathcal{P}$ is finite, there exists a sentence $\xi \in \mathcal{L}$ such that $K=C n(\xi)$; hence, we define $\mathcal{L}_{K}=\mathcal{L}_{\xi}$. Moreover, $C n_{\mathcal{L}_{x}}(x)$ denotes the deductive closure of $x$ in the sublanguage $\mathcal{L}_{x}$, i.e., $C n_{\mathcal{L}_{x}}(x)=C n(x) \cap \mathcal{L}_{x}$.

Peppas et al. further investigated Parikh's original proposal and concluded that there are, in fact, two distinct interpretations of axiom $(\mathrm{P})$; namely, the weak and the strong version of $(\mathrm{P})$ [27]. For presenting these two versions of $(\mathrm{P})$, consider first the next two conditions $(\mathrm{P} 1)$ and $(\mathrm{P} 2)$ which do not refer to a local revision operator -in (P1), $\overline{\mathcal{L}_{x}}$ denotes the (sub)language built from the propositional variables that do not appear in $\mathcal{L}_{x}$, using the standard Boolean connectives (if there are no propositional variables that do not appear in $\mathcal{L}_{x}$, then $\overline{\mathcal{L}_{x}}$ is empty).

\footnotetext{
${ }^{9}$ It is noteworthy that the problem of relevance in the realm of belief change was first highlighted by Gärdenfors in [15]. Although several interpretations of relevance were discussed in that work, the key criterion considered was the following: "If a belief set $K$ is revised by a sentence $\varphi$, then all sentences in $K$ that are irrelevant to the validity of $\varphi$ should be retained in the revised state of belief".
} 
(P1) If $K=C n(x, y), \mathcal{L}_{x} \cap \mathcal{L}_{y}=\varnothing$, and $\mathcal{L}_{\varphi} \subseteq \mathcal{L}_{x}$, then $(K * \varphi) \cap \overline{\mathcal{L}_{x}}=$ $K \cap \overline{\mathcal{L}_{x}}$.

(P2) If $K=C n(x, y), \mathcal{L}_{x} \cap \mathcal{L}_{y}=\varnothing$, and $\mathcal{L}_{\varphi} \subseteq \mathcal{L}_{x}$, then $(K * \varphi) \cap \mathcal{L}_{x}=$ $(C n(x) * \varphi) \cap \mathcal{L}_{x}$.

Condition (P1) corresponds to the weak version of axiom (P). It essentially states that, if a theory $K$ can be expressed in disjoint sublanguages $\mathcal{L}_{x}$ and $\overline{\mathcal{L}_{x}}$, then the revision of $K$ by an epistemic input that can be formulated within $\mathcal{L}_{x}$ should not affect the $\overline{\mathcal{L}_{x}}$-part of $K$. Appending (P2) to (P1), we get the strong version of axiom $(\mathrm{P})$, according to which the modification of the $\mathcal{L}_{x}$-part of $K$ is not affected by the $\overline{\mathcal{L}_{x}}$-part of it. Therefore, in a sense, strong $(\mathrm{P})$ makes the local revision operator $\diamond$ context-independent. ${ }^{10}$

In the remainder of this section, we introduce the necessary terminology for our subsequent discussion. To this aim, for a subset $Q$ of the set $\mathcal{P}$ of propositional variables, $\mathcal{L}^{Q}$ shall denote the sublanguage of $\mathcal{L}$ defined over $Q$, using the standard Boolean connectives (if $Q$ is empty, then $\mathcal{L}^{Q}$ is empty).

Definition 5.1 (Splittable/Confined Theory). Let $K$ be a theory of $\mathcal{L}$. We shall say that $K$ is splittable iff, for some sentences $x, y \in \mathcal{L}, K=C n(x, y)$ and $\mathcal{L}_{x} \cap \mathcal{L}_{y}=\varnothing$. In the special case where $y$ is a tautology and $\mathcal{L}_{x} \subset \mathcal{L}$, we shall say that theory $K$ is confined (to the sublanguage $\mathcal{L}_{x}$ of $\mathcal{L}$ ) as well.

Essentially, a theory $K$ that is confined to a sublanguage $\mathcal{L}^{\prime}$ of $\mathcal{L}$ splits between $\mathcal{L}^{\prime}$ and $\overline{\mathcal{L}^{\prime}}$, with the $\overline{\mathcal{L}^{\prime}}$ part being trivial. In this case, $K$ knows nothing about $\overline{\mathcal{L}^{\prime}}$. ${ }^{11}$

Definition 5.2 (Theory-Splitting, [23]). Let $K$ be a theory of $\mathcal{L}$, and let $Q=\left\{Q_{1}, Q_{2}, \ldots, Q_{n}\right\}$ be a partition of $\mathcal{P}$; i.e., $\cup Q=\mathcal{P}, Q_{i} \neq \varnothing$, and $Q_{i} \cap Q_{j}=\varnothing$, for all $1 \leqslant i \neq j \leqslant n$. The set $Q$ is a $K$-splitting iff there exist sentences $\varphi_{1} \in \mathcal{L}^{Q_{1}}, \varphi_{2} \in \mathcal{L}^{Q_{2}}, \ldots, \varphi_{n} \in \mathcal{L}^{Q_{n}}$, such that $K=C n\left(\varphi_{1}, \varphi_{2}, \ldots, \varphi_{n}\right)$.

${ }^{10}$ The characterization of both conditions (P1) and (P2) in the realm of all popular constructive models for belief revision can be found in $[3,6]$. For a comprehensive study of important constructive aspects of (P2), the interested reader is referred to [8].

${ }^{11}$ It is assumed that tautologies bear no knowledge. 
Parikh showed that, for every theory $K$, there exists a unique finest $K$-splitting - i.e., one which refines every other $K$-splitting-hereafter denoted by $\mathcal{F}_{K}[23] .^{12}$

Example 5.3 (Theory-Splitting). Suppose that $\mathcal{P}=\{a, b, c, d, e\}$, and let $K$ be a splittable theory of $\mathcal{L}$ such that $K=C n(a, b, c \rightarrow d)$. Then, the finest $K$-splitting is $\mathcal{F}_{K}=\{\{a\},\{b\},\{c, d\},\{e\}\}$. Observe that theory $K$ has no information about propositional variable $e$, as it is confined to the sublanguage $\mathcal{L}^{\{a, b, c, d\}}$.

Definition 5.4 (Theory-Units, $[5,3,6]$ ). Let $K$ be a theory of $\mathcal{L}$ which does not contain only tautologies, and let $\left\{F_{1}, F_{2}, \ldots, F_{n}\right\}$ be the finest $K$-splitting. By the definition of a $K$-splitting, there exist contingent sentences $\chi_{1}, \chi_{2}, \ldots, \chi_{m}$ of $\mathcal{L}$, such that $m \leqslant n, \chi_{1} \in \mathcal{L}^{F_{i_{1}}}, \chi_{2} \in$ $\mathcal{L}^{F_{i_{2}}}, \ldots, \chi_{m} \in \mathcal{L}^{F_{i_{m}}}$, and $K=C n\left(\chi_{1}, \chi_{2}, \ldots, \chi_{m}\right)$. The sentences $\chi_{1}, \chi_{2}, \ldots, \chi_{m}$ are the units of $K$, and the set $\mathcal{U}_{K}=\left\{\chi_{1}, \chi_{2}, \ldots, \chi_{m}\right\}$ is the unit set of $K .{ }^{13}$

It turns out that each unit of a theory $K$ is unique, modulo logical equivalence. Intuitively, the units of a splittable theory $K$ are its "building blocks" which divide $K$ into the refined compartments (theories) $C n\left(\chi_{1}\right), C n\left(\chi_{2}\right), \ldots, C n\left(\chi_{m}\right)$. Hence, there is a unique way to think of theory $K$ as being composed of non-trivial (since units are contingent sentences) disjoint compartments, referring to mutually irrelevant subject matters.

Remark 5.5. Each unit of a theory $K$ corresponds to a unique element of the finest $K$-splitting $\mathcal{F}_{K}$. The converse is true only in case $\mathcal{L}_{K}=\mathcal{L}$ (i.e., when theory $K$ is not confined to a sublanguage of $\mathcal{L}$ ); in the case of a confined theory $K$ (where $m<n$ ), not every element of $\mathcal{F}_{K}$ corresponds to a unit of $K$.

Example 5.6 (Theory-Units, Cont'd Example 5.3). The unit set of theory $K=C n(a, b, c \rightarrow d)$ is $\mathcal{U}_{K}=\{a, b, c \rightarrow d\}$. As Remark 5.5 points out, each unit of $K$ corresponds to a unique element of the finest $K$-splitting

${ }^{12}$ Kourousias and Makinson in [20] extended this result to a language built over infinitely many propositional variables. We recall, moreover, that a partition $Q^{\prime}$ refines another partition $Q$ iff, for every $Q_{i}^{\prime} \in Q^{\prime}$, there exists a $Q_{j} \in Q$, such that $Q_{i}^{\prime} \subseteq Q_{j}$.

${ }^{13}$ The definition of units in $[5,3,6]$ is slightly different; herein, a minor modification is made for ease of presentation. 
$\mathcal{F}_{K}=\{\{a\},\{b\},\{c, d\},\{e\}\}$. Yet, since $K$ is confined to the sublanguage $\mathcal{L}^{\{a, b, c, d\}}$, not every element of $\mathcal{F}_{K}$ corresponds to a unit of $K$.

Lastly, the interesting notion of compartmental coupling is introduced, which, to the best of our knowledge, has not been formalized elsewhere before.

Definition 5.7 (Compartmental Coupling). Let $*$ be an AGM revision function, and let $K$ be a splittable theory of $\mathcal{L}$. We shall say that the *-revision of $K$ by a sentence $\varphi \in \mathcal{L}$ couples compartments of $K$ iff an element of the finest $K * \varphi$-splitting $\mathcal{F}_{K * \varphi}$ contains two distinct propositional variables $a, b \in \mathcal{P}$, which belong to distinct elements $F, F^{\prime}$ of the finest $K$-splitting $\mathcal{F}_{K}$ (i.e., $a \in F$ and $b \in F^{\prime}$ ), and at least one of $F, F^{\prime}$ corresponds to a unit of $K$.

Roughly speaking, the revision of a theory $K$ by an epistemic input $\varphi$ couples compartments of $K$ whenever (parts of) two disjoint (refined) compartments of $K$-at least one of which is non-trivial - have been joined into a single (refined) compartment in the revised state of belief. ${ }^{14}$ Evidently, the coupling of compartments of a theory leads to a change in the structure of that theory.

The next concrete examples illustrate (further) features of the above definitions.

Example 5.8 (Revision With Compartmental Coupling, Cont'd 5.6). Consider a sentence $\varphi=\neg a \vee \neg b$, which contradicts theory $K=$ $C n(a, b, c \rightarrow d)$, and an AGM revision function * that respects the strong version of axiom (P), such that $K * \varphi=C n(\neg a \vee \neg b, c \rightarrow d)$. As $\mathcal{F}_{K * \varphi}=\{\{a, b\},\{c, d\},\{e\}\}$, the $*$-revision of theory $K$ by $\varphi$ couples compartments of $K$; this is because the element $\{a, b\}$ of $\mathcal{F}_{K * \varphi}$ contains the propositional variables $a$ and $b$, which belong to distinct elements $F, F^{\prime}$ of $\mathcal{F}_{K}$, and each one of $F, F^{\prime}$ corresponds to a unit of $K$ (recall that $\left.\mathcal{U}_{K}=\{a, b, c \rightarrow d\}\right)$. Notice, lastly, that, as $*$ respects Parikh's principle and theory $K$ is splittable, the part of $K$ formed by the propositional variables $c, d$ and $e$ remains unaffected during the revision-process.

\footnotetext{
${ }^{14}$ Notice that compartmental coupling is defined in terms of finest splittings of theories; therefore, the compartments involved are, as a matter of fact, refined compartments. Furthermore, since, according to Definition 5.7, at least one of $F, F^{\prime} \in \mathcal{F}_{K}$ corresponds to a unit (i.e., contingent sentence) of the initial theory $K$, it follows that at least one of the coupled (refined) compartments of $K$ is non-trivial.
} 
It is evident from the above example that the revision of a splittable theory by an epistemic input does not necessarily couple every compartment of the theory.

Example 5.9 (Revision With Compartmental Coupling). Let $\mathcal{P}=$ $\{a, b, c, d, e, f\}, K=C n(a \leftrightarrow b, c \leftrightarrow d, e \vee f), H=C n(a \leftrightarrow b, e)$ and $\varphi=$ $(b \vee c) \wedge(\neg e) \wedge(\neg f)$-notice that $\varphi$ contradicts both $K$ and $H$. Consider an AGM revision function $*$ such that $K * \varphi=C n(a, b \vee c, d, \neg e, \neg f)$ and $H * \varphi$ $=C n(b \vee c, \neg e, \neg f)$. Hence, we have that $\mathcal{F}_{K}=\{\{a, b\},\{c, d\},\{e, f\}\}$ and $\mathcal{F}_{K * \varphi}=\{\{a\},\{b, c\},\{d\},\{e\},\{f\}\}$, as well as that $\mathcal{F}_{H}=$ $\{\{a, b\},\{c\},\{d\},\{e\},\{f\}\}$ and $\mathcal{F}_{H * \varphi}=\{\{a\},\{b, c\},\{d\},\{e\},\{f\}\}$. Thus, the $*$-revision of $K$ by $\varphi$ couples compartments of $K$, since the element $\{b, c\}$ of $\mathcal{F}_{K * \varphi}$ contains the propositional variables $b$ and $c$, which belong to distinct elements $F, F^{\prime}$ of $\mathcal{F}_{K}$, and each one of $F, F^{\prime}$ corresponds to a unit of $K$. Furthermore, the $*$-revision of $H$ by $\varphi$ couples compartments of $H$, since the element $\{b, c\}$ of $\mathcal{F}_{H * \varphi}$ contains the propositional variables $b$ and $c$, which belong to distinct elements $F^{\prime \prime}, F^{\prime \prime \prime}$ of $\mathcal{F}_{H}$, respectively, and $F^{\prime \prime}$ (but not $F^{\prime \prime \prime}$ ) corresponds to a unit of $H$-recall that, for compartmental coupling, Definition 5.7 requires that at least one of $F^{\prime \prime}, F^{\prime \prime \prime}$ should correspond to a unit of $H$.

Example 5.10 (Revision Without Compartmental Coupling). Let $\mathcal{P}=$ $\{a, b, c, d\}$ and let $K=C n(a, b)$. Then, the finest $K$-splitting is $\mathcal{F}_{K}=\{\{a\},\{b\},\{c\},\{d\}\}$, and the unit set of $K$ is $\mathcal{U}_{K}=\{a, b\}$. Consider, now, a sentence $\varphi=(\neg a) \wedge(\neg b) \wedge(c \vee d)$, which contradicts $K$, and an AGM revision function $*$ such that $K * \varphi=C n(\neg a, \neg b, c \vee d)$; hence, $\mathcal{F}_{K * \varphi}=\{\{a\},\{b\},\{c, d\}\}$. Notice that the $*$-revision of $K$ by $\varphi$ does not couple compartments of $K$, since, although the element $\{c, d\}$ of $\mathcal{F}_{K * \varphi}$ contains the propositional variables $c$ and $d$, which belong to distinct elements $F, F^{\prime}$ of $\mathcal{F}_{K}$, yet, none of $F, F^{\prime}$ corresponds to a unit of $K$ (as the $\mathcal{L}^{\{c, d\}}$-part of $K$ is trivial).

Coupling of compartments can take place even when the epistemic input is consistent with the initial theory; in that case, revision reduces to expansion, due to postulates $(K * 3)-(K * 4)$. Such a scenario is presented in the subsequent example.

Example 5.11 (Expansion With Compartmental Coupling). Let $\mathcal{P}=$ $\{a, b, c, d\}, K=C n(a \vee b, c \vee d)$ and $\varphi=b \vee c$ - notice that $\varphi$ is 
consistent with $K$. Consider an AGM revision function $*$ such that $K * \varphi=C n(a \vee b, b \vee c, c \vee d)$. Then, $\mathcal{F}_{K}=\{\{a, b\},\{c, d\}\}$ and $\mathcal{F}_{K * \varphi}=\{\{a, b, c, d\}\}$. Thus, the $*$-revision (specifically, $*$-expansion) of $K$ by $\varphi$ couples compartments of $K$.

Having introduced the basic concepts of Belief Revision, we turn, in the following sections, to the main contribution of the present article.

\section{Evolution of splittable theories from an epistemological perspective}

Against the background that has been established so far, this section is devoted to an interpretation of rational belief revision of splittable theories from a Kuhnian perspective. In particular, we suggest an epistemological reading of a number of principal belief-change scenarios. Our aim is not an exhaustive investigation of all possible scenarios, but rather an initiation of a discussion between Kuhn and the central figures of Belief Revision.

To this end, let $K$ be a splittable theory of $\mathcal{L}$ such that $K=C n\left(\chi_{1}, \chi_{2}, \ldots, \chi_{m}\right)$, where sentences $\chi_{1}, \chi_{2}, \ldots, \chi_{m}$ (with $\left.m \geqslant 2\right)$ are the units of $K$, and let $\mathcal{F}_{K}=\left\{F_{1}, F_{2}, \ldots, F_{n}\right\}$ be the finest $K$-splitting. ${ }^{15}$ Theory $K$ shall represent the knowledge of a scientific community seen as a single rational agent. Since $K$ can be expressed in disjoint sublanguages, we may assume that it consists of unrelated refined compartments, referring to different subject matters. Furthermore, let $*$ be an AGM revision function that the scientific community utilizes as a tool for revision, and assume that $*$ satisfies the strong version of axiom $(\mathrm{P})$; i.e., conditions (P1) and (P2).

Given an epistemic input $\varphi$, we first distinguish the two cases according to which

$$
\mathcal{L}_{\varphi} \cap \mathcal{L}_{K}=\varnothing \quad \text { or } \quad \mathcal{L}_{\varphi} \cap \mathcal{L}_{K} \neq \varnothing
$$

In the former case, which is abstractly depicted in Figure 1, theory $K$ is necessarily confined to a sublanguage of $\mathcal{L}$, and the epistemic input $\varphi$ is

${ }^{15}$ We assume that the splittable theory $K$ has at least two units (i.e., $m \geqslant 2$ ), so to avoid the trivial case of a theory that refers to a single subject matter. Recall moreover that, by definition, $m \leqslant n$. 


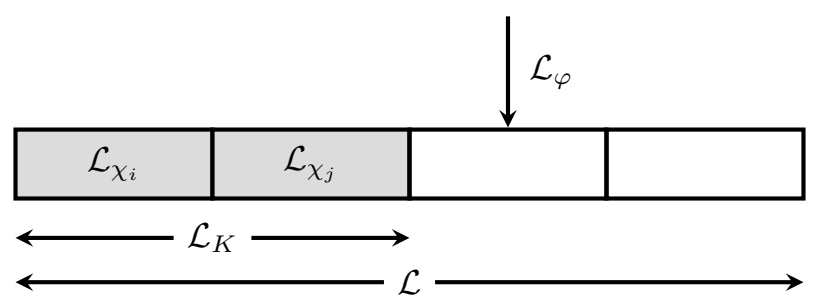

Figure 1. Pre-Paradigm: $K=C n\left(\chi_{i}, \chi_{j}\right)$ and $\mathcal{L}_{\varphi} \cap \mathcal{L}_{K}=\varnothing$. Each block corresponds to an element of the finest $K$-splitting.

clearly consistent with $K$ (since we have assumed that $\varphi$ is self-consistent). Hence, according to the AGM postulates for revision $(K * 1)-(K * 8)$, theory $K$ is (set-theoretically) expanded by $\varphi$; that is,

$$
K * \varphi=K+\varphi .
$$

In this case, a pre-paradigm event takes place, in the context of which new scientific knowledge (that does not contradict initial knowledge) is accumulated, resulting in the formation of new concrete (sub)theories, and, thus, in the establishment of new paradigms.

In the latter case, we further distinguish the two sub-cases according to which $\mathcal{L}_{\varphi}$ is restricted to some sublanguage $\mathcal{L}_{\chi_{i}}$ (with $1 \leqslant i \leqslant m$ ) or not. The former case may be regarded as a period of normal science, whereas, the latter as a paradigm shift. Both these scenarios are formally described in the next two subsections.

\subsection{Normal science: $\quad \mathcal{L}_{\varphi} \subseteq \mathcal{L}_{\chi_{i}} \subseteq \mathcal{L}_{K} \quad$ and $\quad \mathcal{L}_{\varphi} \cap \mathcal{L}_{K} \neq \varnothing$}

During normal science, every new piece of information $\varphi$ is such that its minimal language $\mathcal{L}_{\varphi}$ is a subset of a single sublanguage $\mathcal{L}_{\chi_{i}}$; i.e., $\mathcal{L}_{\varphi} \subseteq$ $\mathcal{L}_{\chi_{i}} \subseteq \mathcal{L}_{K}$ (and, of course, $\mathcal{L}_{\varphi} \cap \mathcal{L}_{K} \neq \varnothing$ ). That is to say, the epistemic input $\varphi$ is related to a single (refined) compartment $C n\left(\chi_{i}\right)$ of $K$, which in turn refers to a specific subject matter - this is abstractly depicted in Figure 2.

In this case, epistemic inputs, which contradict theory $C n\left(\chi_{i}\right)$, correspond to anomalies, that is, challenges to be puzzled out and solved within $C n\left(\chi_{i}\right)$, causing incremental changes. A real-world example of revising 


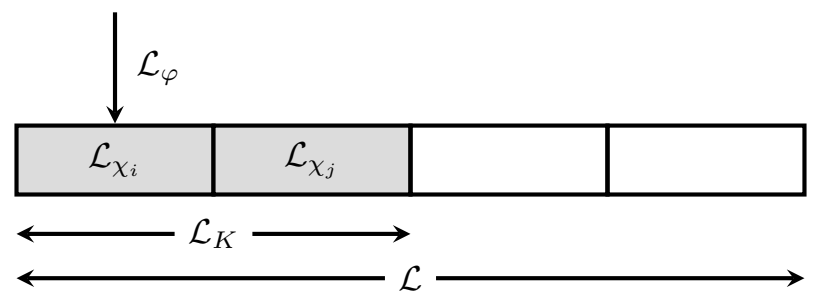

Figure 2. Normal Science: $K=C n\left(\chi_{i}, \chi_{j}\right), \mathcal{L}_{\varphi} \subseteq \mathcal{L}_{\chi_{i}} \subseteq \mathcal{L}_{K}$ and $\mathcal{L}_{\varphi} \cap \mathcal{L}_{K} \neq \varnothing$.

Each block corresponds to an element of the finest $K$-splitting.

a scientific theory by new information, which contradicts previous knowledge, concerns an issue of Cosmology, namely, the expansion of the universe. Specifically, Einstein's static universe, which is a relativistic model of the universe proposed by Albert Einstein in 1917, was refuted after the observations of Edwin Hubble in 1929, suggesting an expanding universe.

On the other hand, new information consistent with theory $C n\left(\chi_{i}\right)$ expands the present knowledge contained in $C n\left(\chi_{i}\right)$, without causing loss of existing information. Such an expansion is constituted by the recent (2015) discovery of gravitational waves, which were predicted in 1916 by Einstein.

In any scenario, under the current hypotheses, conditions $(\mathrm{P} 2)$ and $(\mathrm{F} *)$ entail, for the $\mathcal{L}_{\chi_{i}}$-part of the revised theory $K * \varphi$, that:

$$
(K * \varphi) \cap \mathcal{L}_{\chi_{i}}=\left(C n\left(\chi_{i}\right) * \varphi\right) \cap \mathcal{L}_{\chi_{i}}=\operatorname{th}\left(\min \left([\varphi], \preceq_{C n\left(\chi_{i}\right)}\right)\right) \cap \mathcal{L}_{\chi_{i}} .
$$

Observe that, in order to produce the $\mathcal{L}_{\chi_{i}}$-part of $K * \varphi$, the agent-which can be viewed as the scientific community as a whole - needs only the preorder $\preceq_{C n}\left(\chi_{i}\right)$, and not the preorder $\preceq_{K}$. This, in view of Remark 4.4 of Section 4, allows her to omit comparing the epistemic value of propositions referring to irrelevant subject matters - as the construction of $\preceq_{K}$ demands-, a requirement that would clearly constitute an epistemological "thorn". For instance, how can a rational agent compare the epistemic value of propositions expressed in the language $\mathcal{L}_{\chi_{i}}$, referring to the structure of water, with propositions expressed in the language $\mathcal{L}_{\chi_{j}}$ (with $i \neq j$ ), referring to monetary economics, in order to build a faithful preorder $\preceq_{K}$ ? 
As for the $\overline{\mathcal{L}_{\chi_{i}}}$-part of the revised theory $K * \varphi$, of course, it is equal to $K \cap \overline{\mathcal{L}_{\chi_{i}}}$, according to condition (P1). Hence, only the $\varphi$-relevant compartment of theory $K$ is affected by its $*$-revision by $\varphi$. Clearly then, the following remark is true.

Remark 6.1. In normal science, the finest $K * \varphi$-splitting could be identical to the finest $K$-splitting. Furthermore, since we have assumed that the unit set of $K$ contains at least two units (cf. Footnote 15), it follows that the unit set of $K * \varphi$ is, during a period of normal science, non-singleton.

We close this subsection with a formal concrete example - which is a variation of Example 5.9 of Section 5-illustrating a normal-science scenario.

Example 6.2 (Normal Science). Suppose that $\mathcal{P}=\{a, b, c, d, e, f\}$ and $K=C n(a \leftrightarrow b, c \leftrightarrow d, e \vee f) ;$ thus, $\mathcal{U}_{K}=\{a \leftrightarrow b, c \leftrightarrow d, e \vee f\}$. Let $\varphi=(\neg a \wedge b) \vee(a \wedge \neg b)$ and $\psi=a \wedge b$ - notice that $\varphi$ contradicts $K$, whereas, $\psi$ is consistent with $K$. Assume that $*$ is an AGM revision function that respects the strong version of axiom (P), such that $K * \varphi=C n((\neg a \wedge b) \vee(a \wedge \neg b), c \leftrightarrow d, e \vee f)$ and $K * \psi=C n(a, b, c \leftrightarrow d, e \vee f)$. Hence, we have that $\mathcal{F}_{K}=$ $\{\{a, b\},\{c, d\},\{e, f\}\}, \mathcal{F}_{K * \varphi}=\{\{a, b\},\{c, d\},\{e, f\}\}$ and $\mathcal{F}_{K * \psi}=$ $\{\{a\},\{b\},\{c, d\},\{e, f\}\}$.

In the above example, each one of the epistemic inputs $\varphi$ and $\psi$ is solely related to the (refined) compartment $C n(a \leftrightarrow b)$ of the splittable theory $K$, since $\mathcal{L}_{\varphi} \subseteq \mathcal{L}_{a \leftrightarrow b}$ and $\mathcal{L}_{\psi} \subseteq \mathcal{L}_{a \leftrightarrow b}$. Observe that the $*$-revision of $K$ by $\varphi$ results in a revised theory $K * \varphi$ whose finest splitting is identical to the finest $K$-splitting (cf. Remark 6.1); this is not the case for the $*$-revision of $K$ by $\psi$, which leads to a revised theory $K * \psi$ with a different finest splitting. Lastly, as the AGM revision function $*$ respects Parikh's principle, the $\overline{\mathcal{L}_{a \leftrightarrow b}}$-part of $K$ remains unaffected during the revision-process.

\subsection{Paradigm shift: $\quad \mathcal{L}_{\varphi} \nsubseteq \mathcal{L}_{\chi_{i}}, \quad \mathcal{L}_{\varphi} \cap \mathcal{L}_{K} \neq \varnothing \quad$ and compartmental coupling}

In case $\mathcal{L}_{\varphi} \cap \mathcal{L}_{K} \neq \varnothing$, the minimal language $\mathcal{L}_{\varphi}$ of the epistemic input $\varphi$ is not restricted to a sublanguage $\mathcal{L}_{\chi_{i}}$ (i.e., $\mathcal{L}_{\varphi} \nsubseteq \mathcal{L}_{\chi_{i}}$, for all $i$ such that $1 \leqslant i \leqslant m)$, and, moreover, the revision of $K$ by $\varphi$ couples compartments 
of $K$, over which $\mathcal{L}_{\varphi}$ spans, a paradigm shift takes place in the form of a scientific revolution. Note that the coupling of some compartments of theory $K$ is demanded so as to avoid characterizing as a paradigm shift a situation in which $\mathcal{L}_{\varphi}$ spans over multiple (refined) compartments of $K$, and, yet, the finest $K * \varphi$-splitting is identical to the finest $K$-splitting; i.e., $\mathcal{F}_{K * \varphi}=\mathcal{F}_{K}{ }^{16}$ In other words, given that $\mathcal{L}_{\varphi}$ spans over multiple (refined) compartments of $K$, coupling ensures that some of these (or even all) compartments of $K$-at least one of which is non-trivial-have been joined in the revised state of belief. This, in turn, implies the following observation.

Remark 6.3. Contrary to the case of normal science (cf. Remark 6.1), in the context of a paradigm shift, it is always true that $\mathcal{F}_{K * \varphi} \neq \mathcal{F}_{K}$, and the revision in that context changes the structure (of non-trivial compartments) of the initial theory $K$. Furthermore, a paradigm shift may very well lead to revised theories with singleton unit sets (cf. Example 5.11 of Section 5).

Against this background, the new information $\varphi$ corresponds to a challenging anomaly which, in turn, results in a crisis of normal science; this crisis, eventually, leads to a paradigm shift. Formal instances illustrating the current scenario, which is abstractly depicted in Figure 3, are those encoded in Examples 5.8, 5.9 and 5.11 of Section $5 .{ }^{17}$ It should come as no surprise that an expansion, such as that encoded in Example 5.11, could give rise to a paradigm shift, since an expansion of a splittable theory by new knowledge may lead, due to compartmental coupling, to a dramatic change in the structure (of non-trivial compartments) of the theory (although no information is lost during the expansion). It is, also, noteworthy that Example 5.10 of Section 5 does not correspond to a situation of a paradigm shift, since, although the minimal language of the epistemic input in that example spans over multiple (refined) compartments of the initial theory, compartmental coupling (as defined in Definition 5.7) does not take place during revision.

\footnotetext{
${ }^{16}$ Such a scenario is the following: Let $\mathcal{P}=\{a, b\}, K=C n(a, b), \varphi=\neg a \wedge \neg b$, and consider an AGM revision function $*$ such that $K * \varphi=C n(\neg a, \neg b)$. Clearly then, $\mathcal{L}_{\varphi}$ spans over the (refined) compartments $C n(a)$ and $C n(b)$ of $K$, and, at the same time, $\mathcal{F}_{K * \varphi}=\mathcal{F}_{K}=\{\{a\},\{b\}\}$.

${ }^{17}$ Historical examples for this scenario are reported in the next section. Recall, moreover, that Example 5.10 of Section 5 refers to a revision-instance in which no coupling of compartments of the initial theory takes place.
} 


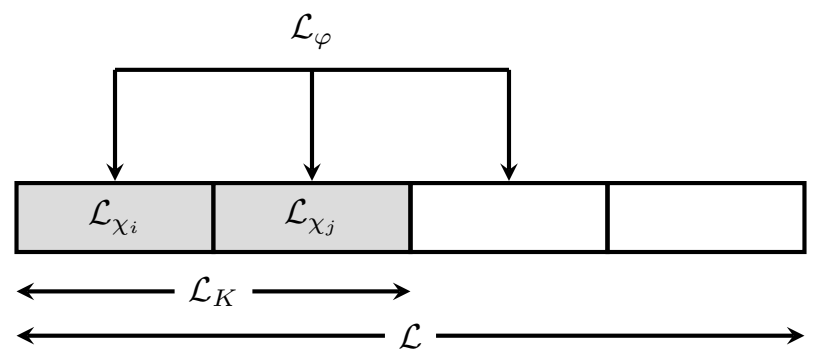

Figure 3. Paradigm Shift: $K=C n\left(\chi_{i}, \chi_{j}\right), \mathcal{L}_{\varphi} \nsubseteq \mathcal{L}_{\chi_{i}}, \mathcal{L}_{\varphi} \cap \mathcal{L}_{K} \neq \varnothing$ and compartmental coupling.

Each block corresponds to an element of the finest $K$-splitting.

\subsection{Observations}

An interesting discussion on the relation between Kuhnian epistemology and (not necessarily relevance-sensitive) belief change has been conducted by Gärdenfors in [14]. In that work, Gärdenfors argues that a paradigm shift, typically, involves a radical change in the epistemic values of the formulae of a scientific theory, and, conversely, a substantial change in the epistemic values of the formulae of a scientific theory is a strong indication of a scientific revolution [14, p. 88]. This change in the epistemic values of formulae is reflected in a change in the faithful preorders that the scientific community assigns to theories, and, as a consequence, in a change of the AGM revision function that the scientific community utilizes for revision. ${ }^{18}$ Such alterations of the revision policy could serve as a means for avoiding "islandings" like that described in Theorem 4.5 (Section 4).

Herein, we supplement the aforementioned view of Gärdenfors by claiming that, in a propositional framework, a scientific revolution could result in a change in the set of propositional variables from which the object language $\mathcal{L}$ is generated, and, vice versa, a change in the set of propositional variables, typically, indicates a scientific revolution. Lastly, a scientific revolution could change the meaning (semantics) of a propositional variable

\footnotetext{
${ }^{18}$ As a matter of fact, a quantification of the difference between faithful preorders is, also, feasible, with the aid of well-accepted concepts such as the Kemeny distance; given any two total preorders over $\mathbb{M}$, their Kemeny distance is defined as the cardinality of their symmetric difference [19].
} 
(for instance, the word "mass" takes a totally different meaning in the Newtonian than in the Einsteinian framework), a fact which is, in turn, related to the symbol grounding problem [17].

We close this section by noting that a formal modelling of the evolution (transition) of scientific theories by means of the AGM paradigm suggests that, contrary to Kuhn's claim (see at the end of Section 2), competing scientific paradigms may be (at least to a certain degree) comparable in a commensurable way, a fact which in turn allows for a rational evaluation among them. ${ }^{19}$ Consider, for example, a scientific community whose knowledge is represented by theory $K_{1}$. Assume that, after a paradigm shift, the knowledge of the scientific community is reflected in a new theory $K_{2}$, whereas, after another paradigm shift, the knowledge of the community is reflected in another new theory $K_{3}$. Against this background, the AGM paradigm provides the formal guidelines in order for an AGM revision function $*$ to be specified, such that, for two sentences $\varphi_{1}, \varphi_{2} \in \mathcal{L}$ (representing new pieces of information), $K_{2}=K_{1} * \varphi_{1}$ and $K_{3}=K_{2} * \varphi_{2} \cdot{ }^{20}$ Since such an AGM revision function $*$ can be defined, the scientific theories of each pair of $K_{1}, K_{2}, K_{3}$ can be compared through $*$, in the sense that one could, for example, generate theory $K_{2}$ from the $*$-revision of $K_{1}$ by $\varphi_{1}$; as earlier stated, this capability allows for a rational evaluation among scientific theories.

\section{Historical examples of paradigm shifts}

Syntheses of initially unrelated scientific theories, after a paradigm shift brought about because a new piece of evidence involved concepts from these theories, have happened in the history of science not just once. Indicative such examples are presented subsequently.

- The theory of magnetism - the formulation of which began with Gilbert's careful study of magnetic phenomena in the late 16th century - was initially unrelated to the theory of electricityformulated, mainly, by Franklin and Coulomb in the late 1700s. The

${ }^{19}$ Although the peculiar nature of the symbol grounding problem involved in a scientific revolution may lead to major difficulties in the comparison.

${ }^{20}$ This is a form of "reverse" belief revision. 
first connection between electric and magnetic phenomena was discovered by Hans Christian Ørsted in 1820, when he found that electric currents produce magnetic forces, namely, a piece of information that involves concepts of magnetism and electricity. Ørsted's discovery was responsible for the formulation of the combined theory of Electromagnetism.

- In the beginning, there was Biology, a discipline that studies life and living organisms, far from Chemistry that studies non-living matter. A dominant principle once biologists believed is imprinted in the following vitalistic view: "Living organisms are fundamentally different from non-living entities because they contain some non-physical element or are governed by different principles than are inanimate things" [10]. Series of strong evidence, however, falsified many vitalistic theories, suggesting that the processes of life are based, in fact, on chemical compounds. That is to say, a paradigm shift associated with Biology led to a new discipline that combines a mixture of both Biology and Chemistry, nowadays called Biochemistry.

- Classical Mechanics depicted a universe in which objects move in perfectly-determined (non-random) ways. In this context, Probability Theory may be considered, at least to a large extent, irrelevant. Ground-breaking experimental discoveries initiated a paradigm shift that led to a new theory of Physics, named Quantum Mechanics, in which the role of pure randomness in physical processes is fundamental.

- Areas of Mathematics such as topology and algebraic geometry, lying at the heart of pure Mathematics and appearing very distant from the Physics frontier, have been dramatically reshaped (in the sense that new discoveries have been emerged) after paradigm shifts associated with fundamental hypotheses of Physics that involved a combination of the aforementioned mathematical principles. This process has led to many hybrid theories, such as the Topological Quantum Field Theory, which now form a core of modern research in both Mathematics and Physics [9]. 
- Connections between two, initially unrelated, theories have not only emerged in the realm of natural sciences. Indicative is the case of Neurobiology and Psychoanalysis, which, at the beginning of the 20th century, seemed completely incompatible. The book by Ansermet and Magistretti, [2], is devoted to the presentation of recent experimental findings - concerning several mechanisms of the brainthat involved a mixture of concepts of both Neurobiology and Psychoanalysis. These observations initiated, in turn, a paradigm shift that revealed a close relation of these two disciplines.

It should be evident that the above points do not refer to arbitrary cases of paradigm shifts, but to cases in which two initially unrelated bodies of knowledge (i.e., compartments of a broader scientific theory) coupled after a new piece of evidence involved concepts of both these bodies. Hence, the presented historical examples respect the hypotheses of the paradigm-shift analysis of Subsection 6.2, and, thus, they come to justify its substance.

\section{Conclusion}

In this article, the evolution (revision) of splittable theories from an epistemological perspective was investigated. In particular, we have suggested an epistemological reading of principal belief-change scenarios, involving splittable belief corpora, from the perspective of Kuhn's influential work on the evolution of scientific knowledge. Representative examples from the history of science have supported the conducted study, providing historical content to the mathematical contours of the introduced concepts. Our analysis aims at the formation of a conceptual bridge between rational belief revision and traditional philosophy of science, which will shed light on the application of formal epistemological tools in the dynamics of (any corpus of) knowledge.

Despite the brevity of the account in the matter of the epistemology of rational belief change, we hope that the ideas conveyed in this article will become the springboard to future research; for instance, other interesting belief-change scenarios could be explored, against the background of Kuhn's principles, or the presented ones could be further refined.

Acknowledgements. I am grateful to Pavlos Peppas and to the anonymous reviewers for their detailed and constructive comments on this article. 


\section{References}

[1] C. Alchourrón, P. Gärdenfors, D. Makinson, On the logic of theory change: Partial meet contraction and revision functions, Journal of Symbolic Logic, vol. 50(2) (1985), pp. 510-530, DOI: https://doi.org/10.2307/ 2274239 .

[2] F. Ansermet, P. Magistretti, Biology of Freedom: Neural Plasticity, Experience, and the Unconscious, Routledge (2007).

[3] T. Aravanis, Relevance and Knowledge Dynamics for Intelligent Agents, Ph.D. thesis, Department of Business Administration, School of Economics \& Business, University of Patras, Patras, Greece (2019).

[4] T. Aravanis, On uniform belief revision, Journal of Logic and Computation, vol. 30 (2020), pp. 1357-1376, DOI: https://doi.org/10.1093/logcom/ exaa058.

[5] T. Aravanis, P. Peppas, M.-A. Williams, Epistemic-entrenchment characterization of Parikh's axiom, [in:] Proceedings of the 26th International Joint Conference on Artificial Intelligence (IJCAI 2017) (2017), pp. 772-778, DOI: https://doi.org/10.24963/ijcai.2017/107.

[6] T. Aravanis, P. Peppas, M.-A. Williams, Full characterization of Parikh's relevance-sensitive axiom for belief revision, Journal of Artificial Intelligence Research, vol. 66 (2019), pp. 765-792, DOI: https://doi.org/10. 1613/jair.1.11838.

[7] T. Aravanis, P. Peppas, M.-A. Williams, An investigation of parametrized difference revision operators, Annals of Mathematics and Artificial Intelligence, vol. 89 (2019), pp. 7-28, DOI: https://doi.org/10.1007/s10472019-09625-x.

[8] T. Aravanis, P. Peppas, M.-A. Williams, A study of possible-worlds semantics of relevance-sensitive belief revision, Journal of Logic and Computation, vol. 30 (2020), pp. 1125-1142, DOI: https://doi.org/10.1093/logcom/ exaa029.

[9] M. Atiyah, R. Dijkgraaf, N. Hitchin, Geometry and physics, Philosophical Transactions of the Royal Society A, vol. 368 (2010), pp. 913-926, DOI: https://doi.org/10.1098/rsta.2009.0227.

[10] W. Bechtel, R. C. Richardson, Vitalism, [in:] E. Craig (ed.), Routledge Encyclopedia of Philosophy, London: Routledge (1998), DOI: https: //doi.org/10.4324/9780415249126-Q109-1. 
[11] G. Brewka, T. Eiter, M. Truszczyński, Answer Set Programming at a glance, Communications of the ACM, vol. 54(12) (2011), pp. 93-103, DOI: https://doi.org/10.1145/2043174.2043195.

[12] M. Dalal, Investigations into theory of knowledge base revision: Preliminary report, [in:] Proceedings of the 7th National Conference of the American Association for Artificial Intelligence (AAAI 1988), The AAAI Press, Menlo Park, California (1988), pp. 475-479.

[13] E. Fermé, S. O. Hansson, Belief Change: Introduction and Overview, Springer International Publishing (2018), DOI: https://doi.org/10.1007/ 978-3-319-60535-7.

[14] P. Gärdenfors, Knowledge in Flux - Modeling the Dynamics of Epistemic States, MIT Press, Cambridge, Massachusetts (1988).

[15] P. Gärdenfors, Belief revision and relevance, [in:] PSA: Proceedings of the Biennial Meeting of the Philosophy of Science Association (1990), pp. 349-365, DOI: https://doi.org/10.2307/193079.

[16] P. Gärdenfors, D. Makinson, Revisions of knowledge systems using epistemic entrenchment, [in:] Proceedings of the 2nd Conference on Theoretical Aspects of Reasoning About Knowledge (TARK 1988), Morgan Kaufmann, Pacific Grove, California (1988), pp. 83-95.

[17] S. Harnad, The symbol grounding problem, Physica D: Nonlinear Phenomena, vol. 42 (1990), pp. 335-346, DOI: https://doi.org/10.1016/01672789(90)90087-6.

[18] H. Katsuno, A. Mendelzon, Propositional knowledge base revision and minimal change, Artificial Intelligence, vol. 52(3) (1991), pp. 263-294, DOI: https://doi.org/10.1016/0004-3702(91)90069-V.

[19] J. G. Kemeny, Mathematics without numbers, Daedalus, vol. 88(4) (1959), pp. 577-591, URL: https://www.jstor.org/stable/20026529.

[20] G. Kourousias, D. Makinson, Parallel interpolation, splitting, and relevance in belief change, Journal of Symbolic Logic, vol. 72(3) (2007), pp. 9941002, DOI: https://doi.org/10.2178/jsl/1191333851.

[21] T. S. Kuhn, The Structure of Scientific Revolutions, University of Chicago Press, Chicago (1970).

[22] E. J. Olsson, S. Enqvist (eds.), Belief Revision meets Philosophy of Science, Springer, Dordrecht (2011), DOI: https://doi.org/10.1007/978-90481-9609-8. 
[23] R. Parikh, Beliefs, belief revision, and splitting languages, [in:] L. S. Moss, J. Ginzburg, M. de Rijke (eds.), Logic, Language and Computation, vol. 2, CSLI Publications (1999), pp. 266-278.

[24] P. Peppas, Belief revision, [in:] F. van Harmelen, V. Lifschitz, B. Porter (eds.), Handbook of Knowledge Representation, Elsevier Science (2008), pp. 317-359, DOI: https://doi.org/10.1016/S1574-6526(07)03008-8.

[25] P. Peppas, M.-A. Williams, Constructive modellings for theory change, Notre Dame Journal of Formal Logic, vol. 36(1) (1995), pp. 120-133, DOI: https://doi.org/10.1305/ndjfl/1040308831.

[26] P. Peppas, M.-A. Williams, Parametrised difference revision, [in:] Proceedings of the 16th International Conference on Principles of Knowledge Representation and Reasoning (KR 2018) (2018), pp. 277-286.

[27] P. Peppas, M.-A. Williams, S. Chopra, N. Foo, Relevance in belief revision, Artificial Intelligence, vol. 229 (2015), pp. 126-138, DOI: https://doi. org/10.1016/j.artint.2015.08.007.

\section{Theofanis Aravanis}

University of Patras

School of Economics \& Business

Department of Business Administration

Patras 265 00, Greece

University of the Peloponnese

School of Engineering

Department of Mechanical Engineering

Patras 263 34, Greece

e-mail: taravanis@upatras.gr 